\title{
Pattern formation in a spatial plant-wrack model with tide effect on the wrack
}

\author{
Gui-Quan Sun · Li Li • Zhen Jin • Bai-Lian Li
}

Received: 9 March 2009 / Accepted: 12 May 2009 /

Published online: 30 June 2009

(C) The Author(s) 2009. This article is published with open access at Springerlink.com

\begin{abstract}
Spatial patterns are a subfield of spatial ecology, and these patterns modify the temporal dynamics and stability properties of population densities at a range of spatial scales. Localized ecological interactions can generate striking large-scale spatial patterns in ecosystems through spatial self-organization. Possible mechanisms include oscillating consumer-resource interactions, localized disturbance-recovery processes, and scale-dependent feedback. However, in this paper, our main aim is to study the effect of tide on the pattern formation of a spatial plant-wrack model. We discuss the changes of the wavelength, wave speed, and the conditions of the spatial pattern formation, according to the dispersion relation formula. Both the mathematical analysis and numerical simulations reveal that the tide has great influence on the spatial pattern. More specifically, typical traveling spatial patterns can be obtained. Our obtained results are consistent with the previous observation that wracks exhibit traveling patterns, which is useful to help us better understand the dynamics of the real ecosystems.
\end{abstract}

Keywords Carex stricta $\cdot$ Pattern formation • Tide

G.-Q. Sun $\cdot$ L. Li $\cdot$ Z. Jin $(\bowtie)$

Department of Mathematics, North University of China, Taiyuan, Shan'xi 030051, People's Republic of China e-mail: jinzhn@263.net

L. Li

e-mail: 1ili831113@163.com

G.-Q. Sun

School of Mechatronic Engineering, North University of China, Taiyuan, Shan'xi 030051, People's Republic of China e-mail: gquansun@yahoo.com.cn

G.-Q. Sun · B.-L. Li

Ecological Complexity and Modeling Laboratory, Department of Botany and Plant Sciences, University of California, Riverside, CA 92521-0124, USA

B.-L. Li

e-mail: bai-lian.li@ucr.edu 


\section{Introduction}

The study of complexity in ecosystems has been recognized as one of the frontiers of ecology [1]. Many ecosystems exhibit remarkable complexity in both structure and dynamics, which has repeatedly frustrated attempts to predict the response of ecosystems to human interference [2]. Complexity within ecosystems, however, has also been recognized as the basis for the adaptive capacity of ecosystems to future changes on both regional and global scales [3-5]. A key property of complex systems is emergence, where properties of the ecosystem at large spatial scales result from feedback interactions between components occurring at smaller scales $[5,6]$. These emergent properties result from the capacity of systems to self-organize [5, 7-31].

More recently, many ecologists have paid more and more attention to the experimental investigation of regular spatial patterning in Carex stricta. Carex stricta, the tussock sedge, is a species with widespread distribution in freshwater marshes of North America. Spatial dispersals of vegetation (through tillers) and wrack (resulting from dead plant leaves dropping to the soil surface and movement by the tides) are modeled using a diffusion approximation. The model, which describes the interaction of the plant and wrack, is as follows [32]:

$$
\begin{gathered}
\frac{\partial P}{d t}=P(1-P) F(P)-s P-I(P, W)+d_{P} \Delta P, \\
\frac{\partial W}{d t}=s P-b W+d_{W} \Delta W,
\end{gathered}
$$

where $P$ is plant the biomass, $W$ is the wrack biomass, $F(P)$ is a function describing the positive effect of plant biomass on its own growth, $s$ is the specific rate of plant senescence, $I(P, W)$ is a function describing the inhibiting effect of wrack on plant growth as a function of plant and the wrack biomass, $b$ is the decay rate of wrack, and $d_{P}$ and $d_{W}$ are diffusion constants describing lateral movement of plants and wrack. Here, $\Delta=\partial^{2} / \partial X^{2}+\partial^{2} / \partial Y^{2}$ is the usual Laplacian operator in two-dimensional space.

However, another type of dynamics will emerge when the mobility of the tides exhibits a correlated motion towards a certain direction, which is the cyclic rising and falling of Earth's ocean surface caused by the tidal forces of the Moon and the Sun acting on the oceans. Tides are important natural forces that cause changes in the depth of marine and estuarine water bodies and produce oscillating currents (see http://en.wikipedia.org/wiki/Tide for more detail), which may have great impact on the spatial patterns of plant and wrack population dynamics.

In the present paper, we want to see the effect of tidal current on the pattern formation of both the plant and wrack. To exclude the Turing pattern, we set $d_{P}=d_{W}=1$. Additionally, system (1) will be changed as:

$$
\begin{gathered}
\frac{\partial P}{d t}=P(1-P) F(P)-s P-I(P, W)+\Delta P, \\
\frac{\partial W}{d t}=s P-b W+\Delta W+\delta \nabla W .
\end{gathered}
$$

Advection by tidal current is represented by the gradient operator $\nabla W$ multiplied by the advection constant $\delta$ [23]. For this simple model, we assume that tide only has an effect on the wrack for the reason that the wrack is much lighter than the plants in weight. 


\section{Mathematical analysis}

In this model, we focus on the following specific type with an indirect facilitation of growth by the root mound by lowering of inhibition by the wrack, which leads to:

$$
\begin{gathered}
F(P)=1, \\
I(P, W)=a P W K /(P+K),
\end{gathered}
$$

where $K /(P+K)$ is added to the inhibition term, lowering inhibition as $P$ increases, $K$ is the level of plant biomass where inhibition is lowered by half, and $a$ is an inhibition coefficient [32]. As a result, kinetics of the system are

$$
\begin{gathered}
f(P, W)=P(1-P)-s P-a P W \frac{K}{P+K}, \\
g(P, W)=s P-b W .
\end{gathered}
$$

In order to provide guidelines on the dynamics of the full reaction-diffusion-advection system, it is important to consider the local dynamics of the system [33]. It is, naturally, the dynamics in the biologically meaningful region $P \geq 0, W \geq 0$ that are of interest. By considering the nullclines $f=0, g=0$, and the intersection of these curves in phase space, linear stability analysis reveals that we have a stationary point at $(0,0)$ for the kinetics. The stability is determined by $s$, i.e., it is stable if $s>1$ and unstable if $s<1$ (which is a saddle). Furthermore, we want to find the positive steady state of the non-spatial model, $\left(P^{*}, W^{*}\right)$, which corresponds to the coexistence of plant and wrack. By direct calculation, we find that, if $s<1$, there exists a unique positive steady state, and

$$
\begin{gathered}
P^{*}=\frac{b-a s K-K b-s b+\sqrt{M}}{2 b}, \\
W^{*}=\frac{s P^{*}}{b},
\end{gathered}
$$

where $\quad M=b^{2}-2 a b s K+2 K b^{2}-2 s b^{2}+a^{2} s^{2} K^{2}+2 a s b K^{2}+2 a s^{2} K b+K^{2} b^{2}-2 K b^{2} s+$ $s^{2} b^{2}$. Additionally, if $s>1$, there is no positive steady state.

From the biological point of view, we are interested in studying the stability behavior of the positive steady state $\left(P^{*}, W^{*}\right)$. The Jacobian corresponding to this equilibrium point is

$$
J=\left(\begin{array}{ll}
a_{11} & a_{12} \\
a_{21} & a_{22}
\end{array}\right),
$$

where the elements are the partial derivatives of the reaction kinetics evaluated at the stationary state $\left(P^{*}, W^{*}\right)$. For the sake of brevity, we do not write down the expression for the elements.

It is easy to see that model (2) has the form of a generalized reaction-diffusionadvection model. To consider pattern formation for system (2), we should look for the dispersion relation, which gives the condition for pattern formation. Following [34], we will assume periodic (or zero-flux) boundary conditions and prescribed initial conditions. A symmetry breaking occurs when a homogeneous steady state solution of system (2) is linearly stable to perturbations in the absence of the diffusion and advection terms, but linearly unstable to small spatial perturbations in the presence of diffusion and advection. 
It is a simple matter to find the exact algebraic formulation of the dispersion relation when these equations are subject to certain boundary conditions and stability properties. Here, we consider an approach based on Laplace transform methods, which is convenient for finding symmetry breaking (Turing instability) conditions in the case when the systems include diffusion and advection. The Laplace transform methods are extensively used in the reaction-advection-diffusion equations including the system with fractional diffusion and fractional reaction [35-38].

We make the following substitution:

$$
P=P^{*}+\bar{P}(\vec{r}, t),
$$

and

$$
W=W^{*}+\bar{W}(\vec{r}, t)
$$

into the kinetic equations and assume $|\bar{P}|,|\bar{W}| \ll 1$. Then, in the linear approximation, we have

$$
\begin{gathered}
\frac{\partial \bar{P}}{\partial t}=a_{11} \bar{P}+a_{12} \bar{I}+\nabla^{2} \bar{P} \\
\frac{\partial \bar{W}}{\partial t}=a_{21} \bar{W}+a_{22} \bar{W}+\nabla^{2} \bar{W}+\delta \nabla \bar{W} .
\end{gathered}
$$

The initial conditions are assumed as

$$
\left.\bar{P}\right|_{t=0}=h(\vec{r})
$$

and

$$
\left.\bar{W}\right|_{t=0}=z(\vec{r}),
$$

where the functions $h(\vec{r})$ and $z(\vec{r})$ decay rapidly for $\vec{r} \rightarrow \pm \infty$. Following the standard approach, let us now perform a Laplace transformation of the linearized equations over the two independent variables $\vec{r}$ and $t$. For $\vec{r}$, we use the so-called two-sided version of the transformation. The relations for the forward and backward transforms are

$$
P_{s q}=\int_{0}^{\infty} e^{-s t} d t \int_{-\infty}^{+\infty} \bar{P}(\vec{r}, t) e^{-q \vec{r}} d \vec{r}
$$

and

$$
\bar{P}(\vec{r}, t)=-\frac{1}{4 \pi^{2}} \int_{\beta-i \infty}^{\beta+i \infty} e^{s t} d s \int_{-i \infty}^{i \infty} P_{s q} e^{q \vec{r}} d q,
$$

where $s$ and $q$ are complex variables. Additionally, $s$ is the Laplace transform variable and $q$ is the Fourier transform variable. That is to say that $q=i k$, or $(i k, i l)$, corresponding to one- and two-dimensional space, and the wave numbers $k$ and $l$ are real numbers. In formula (10), for the backward transformation, the integration contour in the $q$ plane is the imaginary axis. In the $s$ plane, the contour is parallel to the imaginary axis and located to the right of all singularities of the integrand.

After this transformation, the kinetic equations read

$$
\left(s-a_{11}-q^{2}\right) P_{s q}-a_{12} W_{s q}=H(q)
$$


and

$$
\left(s-a_{22}-\delta q-q^{2}\right) W_{s q}-a_{21} P_{s q}=Z(q),
$$

where $H(q)$ and $Z(q)$ are the transforms of $h(\vec{r})$ and $z(\vec{r})$. To reveal the presence of an instability and disclose its character, it is sufficient to consider one variable. The temporal growth of the perturbations can now be found by inverting the Laplace transforms, which follows directly after factorizing the denominator. In the presence of advection, the instability is either absolute or convective [39-45]. If the advection coefficient is small, the diffusion carries the perturbation upstream faster than the advection sweeps. As a result, the perturbation invades the whole space. This situation is referred to as an absolute instability. If the advection coefficient is large, the advection acts faster than the diffusions do. Therefore, the perturbation asymptotically decays towards zero at every fixed point. This is the case of the convective instability. The transition between these two cases happens at the critical advection coefficient that can be determined from the set of equations [37, 46, 47] (see also references therein):

$$
D(s, q)=0 .
$$

By solving the linear equations (13) and (14), we find $P_{s q}$ and then use the backward transformation (12) to obtain the following formal solution:

$$
\bar{P}(\vec{r}, t)=-\frac{1}{4 \pi^{2}} \int_{\beta-i \infty}^{\beta+i \infty} e^{s t} d s \int_{-i \infty}^{i \infty} \frac{\left(s-a_{22}+\delta q-q^{2}\right) F(q)+a_{12} G(q)}{D(s, q)} e^{q \vec{r}} d q .
$$

Then, we obtain the linear stability of this state, which is described by the dispersion relation

$$
D(s, q)=\left(s-a_{22}-\delta q-q^{2}\right)\left(s-a_{11}-q^{2}\right)-a_{12} a_{21} .
$$

For the sake of convenience, we first consider to the one-dimensional case. By setting $q=$ $i k$, we have that

$$
s=\frac{a_{11}+a_{22}-2 k^{2}}{2}+\frac{\delta k i}{2} \pm \frac{\sqrt{A+B i}}{2}
$$

where

$$
A=a_{11}^{2}-2 a_{11} a_{22}+a_{22}^{2}-\delta^{2} k^{2}+4 a_{12} a_{21},
$$

and

$$
B=-2 a_{11} \delta k+2 a_{22} \delta k .
$$

Straightforward manipulation of (17) yields

$$
\operatorname{Re}(s)=\frac{a_{11}+a_{22}-2 k^{2}}{2} \pm \frac{1}{2} \sqrt{\frac{1}{2}\left(\sqrt{A^{2}+B^{2}}+A\right)}
$$

and

$$
\operatorname{Im}(s)=\frac{\delta k i}{2} \pm \operatorname{sign}(B) \frac{1}{2} \sqrt{\frac{1}{2}\left(\sqrt{A^{2}+B^{2}}-A\right)} .
$$


Under these conditions, the spatial mode $q$ (in one- or two-dimensional space) is unstable, and thus, system (2) grows into a pattern, that is, $\operatorname{Re}(\lambda)>0$. For the non-spatial model, the equilibria should be locally stable, i.e., $a_{11}+a_{22}<0$ and $a_{11} a_{22}-a_{12} a_{21}>0$. By direct calculation, we find that the condition for emergent pattern formation is

$$
\delta>\frac{\left(-a_{11}-a_{22}+2 k^{2}\right) \sqrt{-\left(a_{11} a_{22}-a_{22} k^{2}+k^{4}\right) \Theta}}{\left(a_{11} a_{22}-a_{22} k^{2}-a_{11} k^{2}+k^{4}\right) k},
$$

where

$$
\Theta=\left(k^{4}-a_{22} k^{2}-a_{11} k^{2}-a_{12} a_{21}+a_{11} a_{22}\right) .
$$

\section{Numerical results}

The dynamical behavior of the spatial model cannot be studied by using analytical methods or normal forms. Thus, we have to perform simulations by computer. In this section, we give the numerical simulations of the partial differential equations (PDEs) (2) in the oneand two-dimensional space. We have taken the approach of discretizing the PDEs in space, applying upwinding to the convective term and Euler integration of the finite difference equations, which means approximating the value of the concentration at the next time step based on the rate of change of the concentration at the previous time step. Here, the time stepsize $\Delta t=0.05$ and space stepsize (lattice constant) $\Delta h=1$. The scale of the space and time is averaged for the Euler method. We used a rectangular spatial grid consisting of $50 \times 50$ cells, with a unidirectional water flow in the $y$ axis direction.

All our numerical simulations employ periodic (zero-flux) boundary conditions. As far as we know, there are two different initial conditions used for analysis of the spatial extended systems [48-51]. The first one, which is more general from the biological point of view, is random spatial distribution of the species. The other one is a special choice, which is made by taking the species community in a horizontal layer as decreasing gradually and the vertical distribution of species as homogeneous. In this paper, we choose the first method. The initial density distribution corresponds to random perturbations around the trivial stationary state $\left(P^{*}, W^{*}\right)$ in model (2), with a variance significantly lower than the amplitude of the final patterns, which seems to be more general from the biological point of view.

In most cases, we used the explicit scheme. In order to avoid numerical artifacts, we checked the sensitivity of the results to the choice of the time and space steps, and their values have been chosen as sufficiently small. Also, some of the results were reproduced by means of using a more advanced alternate directions scheme. Both numerical schemes are standard; hence, we do not describe them here; details and particulars can be found in [52].

It must be mentioned that, since the system under study depends on a relatively large number of parameters (five for the system (2)), its detailed numerical investigation in the whole parameter space is virtually impossible. Instead, we choose one controlling parameter and consider the changes in the pattern formation subject to its variation. In this paper, we choose the advection coefficient $\delta$ as the controlling factor and keep all other parameters fixed. The choice of $\delta$ as the controlling factor is justified by the results of field observations, showing that intensity of advection affects the pattern formation. Also, this choice is consistent with our objective in this paper, i.e., finding the effect of the advection on the regular pattern formation in freshwater systems. 
Figure 1 shows the evolution of the spatial pattern of both plant and wrack at $t=385$, 490, and 705, with small random perturbation of the stationary solution $P^{*}$ and $W^{*}$ of the spatially homogeneous systems when the parameters can ensure $\operatorname{Re}(\lambda)>0$. In this case, one can see that there are travelling spatial patterns with $\delta=2.5$. In Fig. 2, we show the typical spatial pattern formation of both the plant and wrack with $\delta=4$, which is similar to Fig. 1. However, the wavelength in Fig. 2 is larger than that in Fig. 1. That is to say, the single parameter of the system (3), namely, $\delta$, can lead to dramatic changes in the qualitative

a

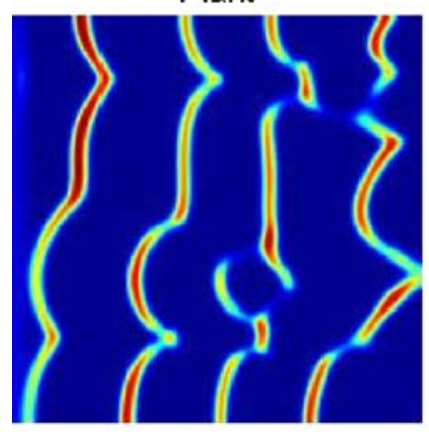

b

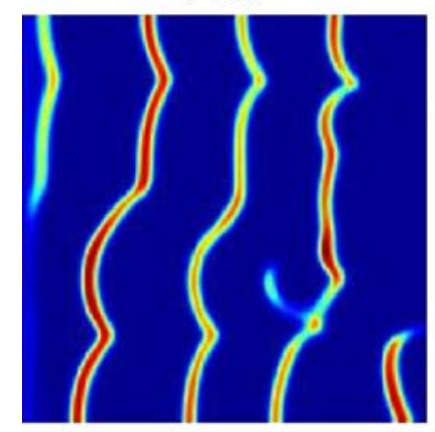

C

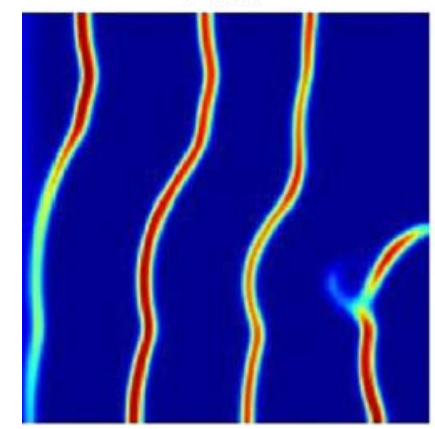

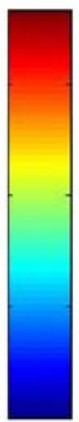

0.3

0.2

0.1

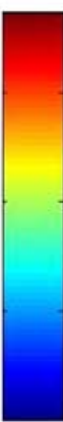

0.3

0.2

0.1

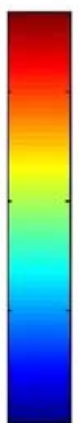

Wrack
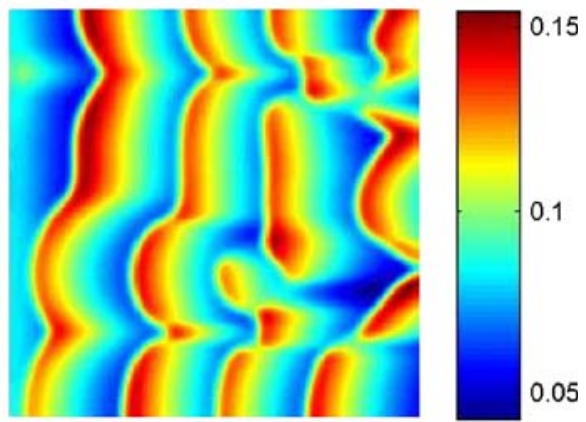

Wrack

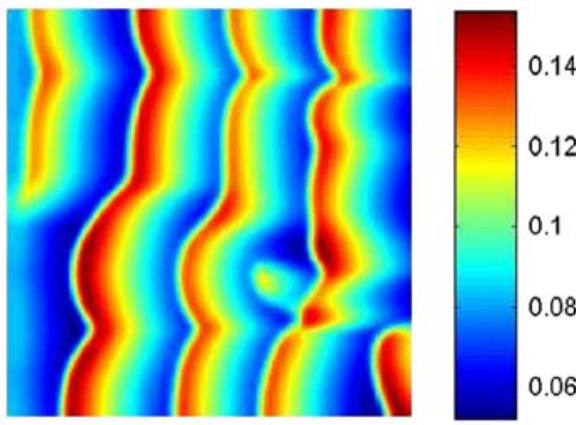

Wrack
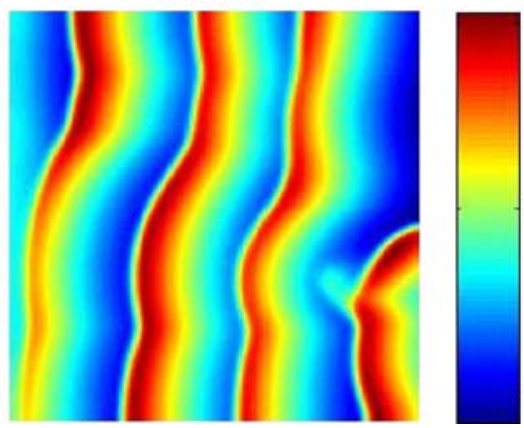

0.15

Fig. 1 Snapshots of contour pictures, showing the time evolution of the density of both plant and wrack at different instants with $\delta=2.5$. Other parameter values are: $s=0.25, b=0.1, a=10$, and $K=0.2$. $\mathbf{a} t=385, \mathbf{b} t=490, \mathbf{c} t=705$ 
a
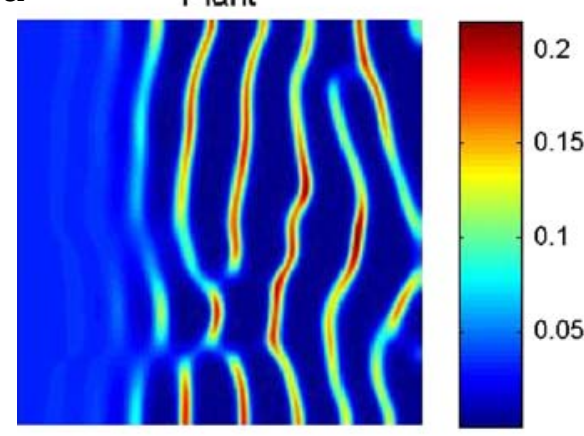

b
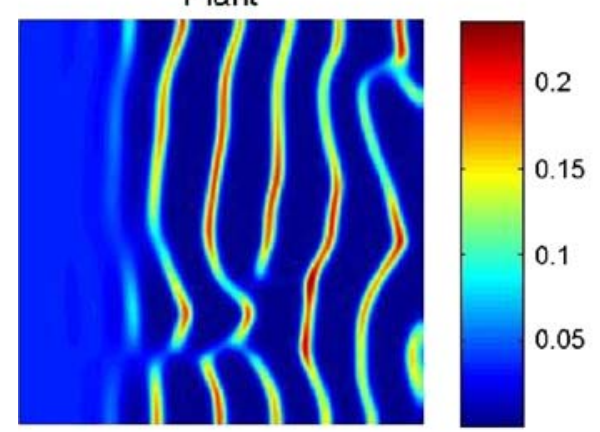

C

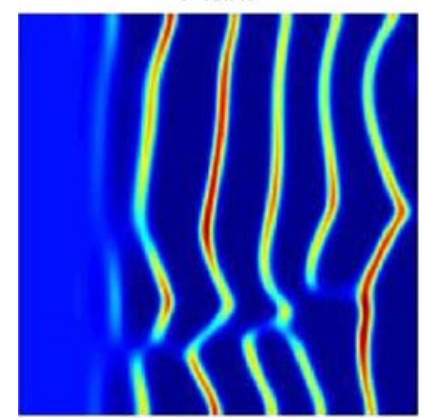

Plant

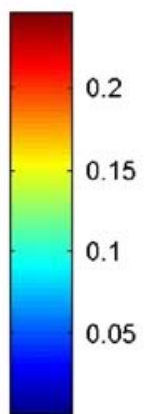

Wrack
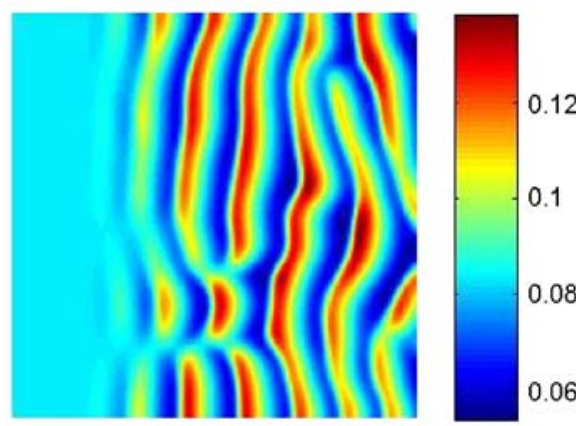

Wrack

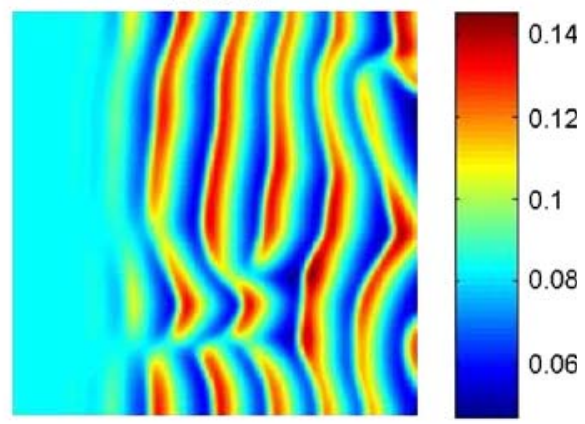

Wrack

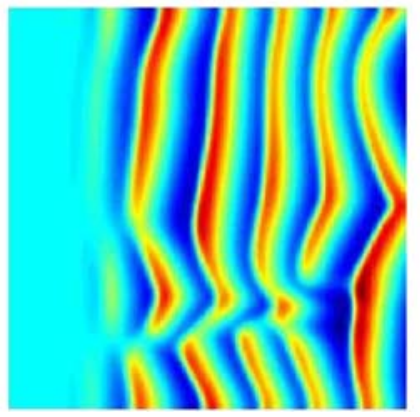

Fig. 2 Snapshots of contour pictures, showing the time evolution of the density of both plant and wrack at different instants with $\delta=4$. Other parameter values are: $s=0.25, b=0.1, a=10$, and $K=0.2$. a $t=385$, $\mathbf{b} t=490, \mathbf{c} t=705$

dynamics of the solutions. The parameter $\delta$ is the coefficient of the tidal current. From the biological point of view, how fast the tide diffuses can have a profound effect on the fate of the system.

In order to clearly see the evolution of the pattern, we show spatial plots in Fig. 3. It is well known that the basic arguments in spiral stability analysis can be carried out by reducing the system to one-dimensional space [53-56]. The method of generating space-time plots is to let $Y$ be a constant from each pattern snapshot (here, $Y=25$, the 

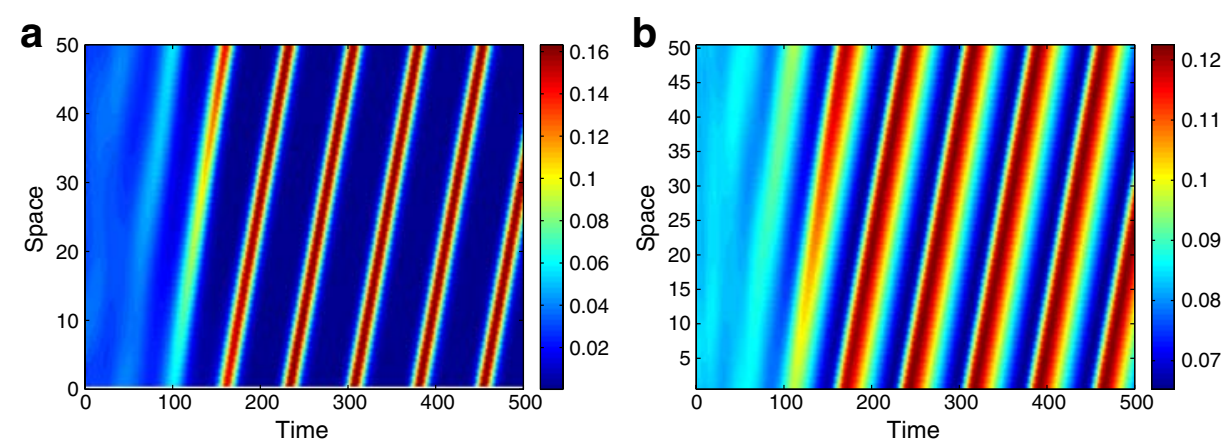

Fig. 3 Typical spatiotemporal pattern diagram for model (2) with the advection turning on. a The pattern of the plant. b The pattern of the wrack. Here, the parameters are $s=0.25, b=0.1, a=10, K=0.2$, and $\delta=4$

corresponding to the center line), and to stack these lines in temporal order. As seen from Fig. 3, a typical traveling spatial pattern is shown, which is consistent with Fig. 2.

\section{Properties of traveling wave solutions}

Our numerical analysis suggests that system (2) does not have any stationary patterns, with the homogeneous steady states being the only stationary solution. Instead, the patterns move at a constant speed, which means that the patterns are a periodic traveling wave solution. For the sake of convenience, we first investigate patterns in the one-dimensional space, which have the mathematical form $P(X, t)=P(\xi)$ and $W(X, t)=W(\xi)$, where $\xi=X+c t$ and $c$ is the migration speed. The results are also valid in two-dimensional space. Substituting these solution forms into (3) gives the ordinary differential equations

$$
\begin{gathered}
P(1-P)-s P-\frac{a P W K}{P+K}+c \frac{d P}{d \xi}+\frac{d^{2} P}{d \xi^{2}}=0, \\
s P-b W+(\delta+c) \frac{d W}{d \xi}+\frac{d^{2} W}{d \xi^{2}}=0,
\end{gathered}
$$

where $\xi$ is now treated as a time- or space-like variable. This second-order ordinary differential equation predicts spatial periodic wave forms. Equation 25 can then be written as a system of four first-order ordinary differential equations, which are as follows:

$$
\begin{gathered}
\frac{d P}{d \xi}=u \\
\frac{d u}{d \xi}=-P(1-P)+s P+\frac{a P W K}{P+K}-c u \\
\frac{d W}{d \xi}=v \\
\frac{d v}{d \xi}=-s P+b W-(\delta+c) v
\end{gathered}
$$



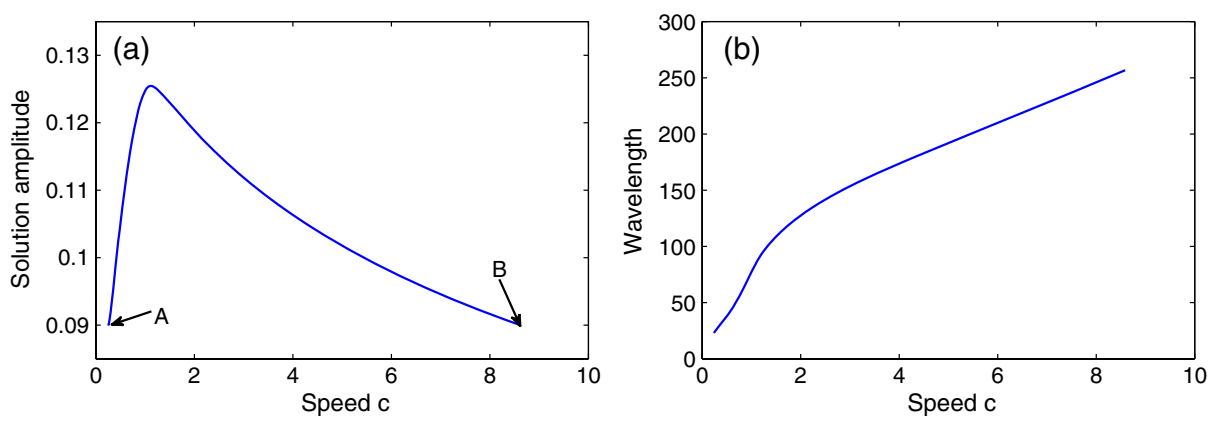

Fig. 4 Typical bifurcation diagrams for the ordinary differential equations (26). a The changes in stability occur via Hopf bifurcations, from which arise branches of periodic orbits, which are marked $A$ and $B$. b Wavelength along the periodic orbit branches. Here, the parameters are $s=0.25, b=0.1, a=10, K=0.2$, and $\delta=4$

It is easy to obtain the steady-state solutions to this system, which are of the form $(P, u, W, v)=\left(P^{*}, 0, W^{*}, 0\right)$, where $\left(P^{*}, W^{*}\right)$ is the spatially uniform steady-state solution of the nonspatial equation (2).

In the following, we track the family of traveling wave solutions of (26) by using the software package AUTO [57], which is a software tool partly designed for continuation and bifurcation problems in ordinary differential equations. We can use it to locate a period of that periodic traveling wave that varies as we gradually change the equation parameters. The first calculation is to locate the wave speed at which there is a Hopf bifurcation in (26), and the starting point is the homogeneous steady state (26).

We plot the typical bifurcation diagrams in Fig. 4, which shows that the changes of stability occur via Hopf bifurcations with respect to the speed $c$, from which arises a single branch that links the two Hopf points. When the speed is above the critical value of speed, the stability of the equilibrium will be lost and a periodic orbit is born

The bifurcation diagrams of solution amplitudes are represented in Fig. 5, where we use the standard definition of $L_{2}$-norm

$$
L_{2}=\sqrt{\frac{\int_{0}^{L} \sum_{k=1}^{4} V_{k}^{2}(\xi) d \xi}{L}},
$$
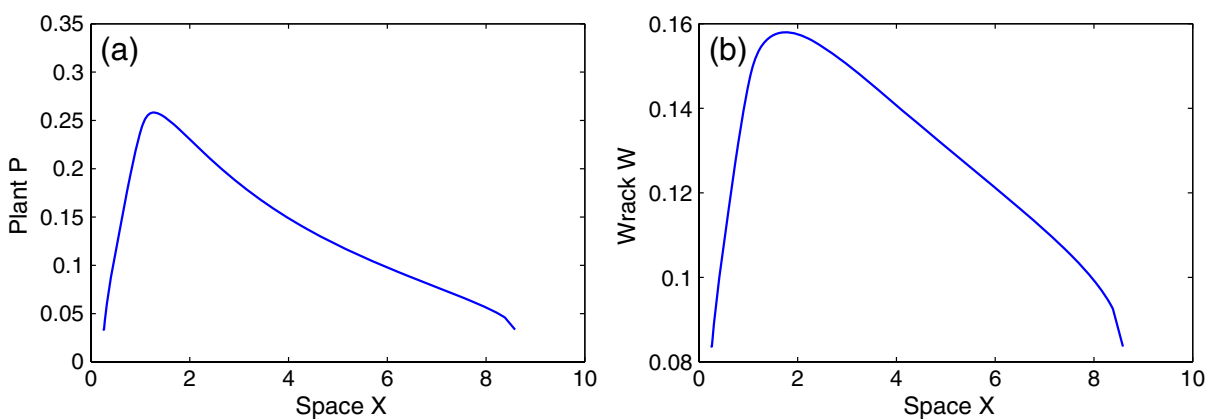

Fig. 5 Snapshots of spatial periodic travelling solution within unit period/wavelenght. Here, the parameters are $s=0.25, b=0.1, a=10, K=0.2$, and $\delta=4$ 

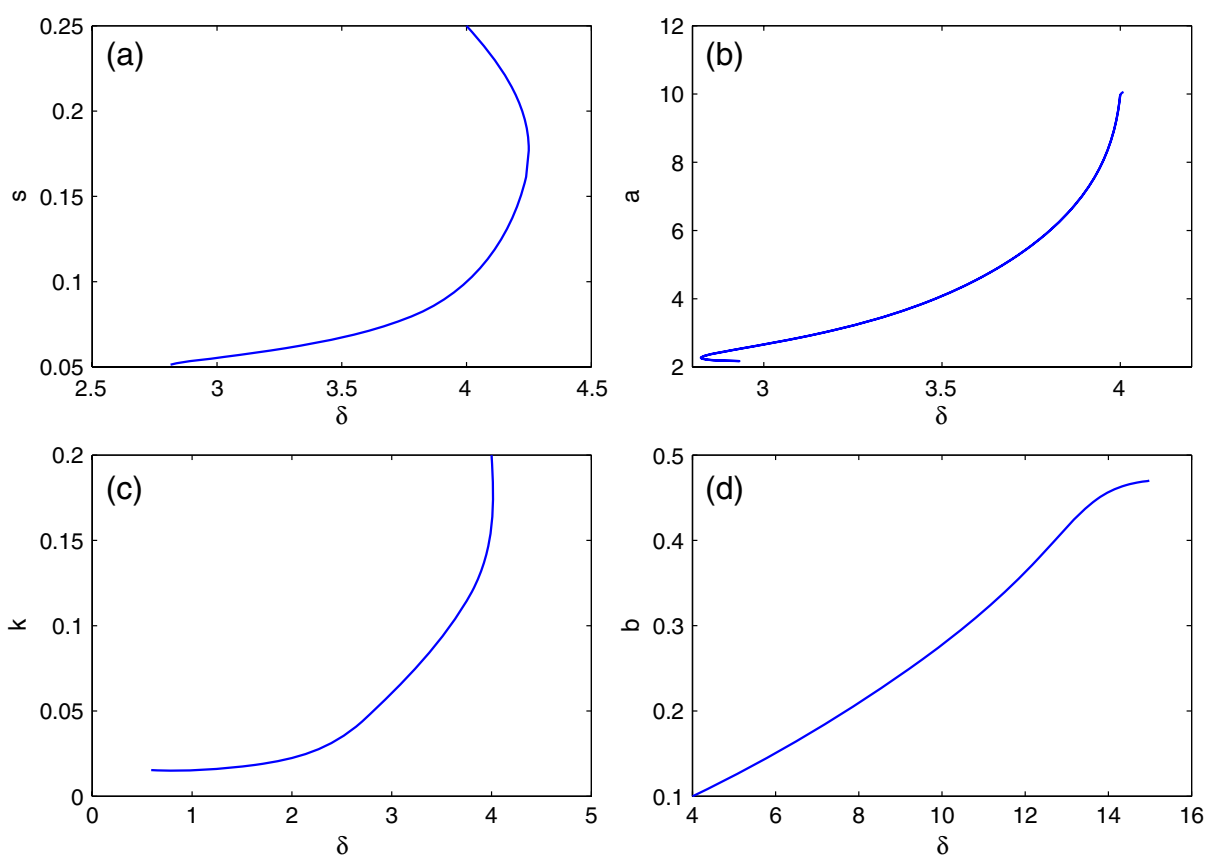

Fig. 6 An illustration of the variations in parameter space of the solution structure of the ordinary differential equations (26). We plot the loci of Hopf bifurcation lines and patterns of fixed wavelength in a $\delta$ vs $s, \mathbf{b} \delta$ vs $a$, c $\delta$ vs $K$, and $\mathbf{d} \delta$ vs $b$. Here, the parameters are $s=0.25, b=0.1, a=10, K=0.2$, and $c=4$

where $L$ is the spatial period and $V_{k}(\xi)$ are four variables of four first-order ordinary differential equations about (26). Figure 5 shows that the periodic orbits are formed by bifurcation with an intermediate range of speeds.

From the practical point of view, one may also be concerned with the effect of other parameters, such as $s, a, K$, and $b$. Using AUTO, it is possible to track the locus of the Hopf bifurcation lines, which we show in Fig. 6.

\section{Discussion and conclusion}

In this paper, we investigate spatial pattern formation in a plant-wrack model combined with tide effects. By obtaining the dispersion relation by numerical calculation, we discuss the effects of tide on the dispersion relation including the wavelength, and conditions of spatial pattern formation by using linear analysis. Furthermore, we perform nonlinear analysis by using the bifurcation package AUTO to confirm our numerical prediction resulting from the linear analysis.

In [32], they investigate the plant-wrack model only with the diffusion term, and obtain typical Turing patterns. However, when the former system is combined with the tidal current term, typical travelling patterns emerge. In the real world, wrack exhibits travelling patterns $[58,59]$. That is to say, our obtained results are consistent with the real world. 
Large-scale spatial patterns widely exist inecosystems. Despite abundant theoretical literature, studies revealing spatial self-organization in real ecosystems are very few [23-27]. Further research is necessary to understand and predict regular pattern formation in ecosystems [60].

Acknowledgements This work is supported by the National Natural Science Foundation of China under Grant No. 60771026, Program for New Century Excellent Talents in University (NCET050271), the Special Scientific Research Foundation for the Subjects of Doctors in University (20060110005), Graduate Students' Excellent Innovative Item of Shanxi Province No. 20081018, and the US National Science Foundation BioComplexity Program and the University of California Agricultural Experiment Station.

Open Access This article is distributed under the terms of the Creative Commons Attribution Noncommercial License which permits any noncommercial use, distribution, and reproduction in any medium, provided the original author(s) and source are credited.

\section{References}

1. Thompson, J.N., Reichman, O.J., Morin, P.J., Polis, G.A., Power, M.E., Sterner, R.W., Couch, C.A., Gough, L., Holt, R., Hooper, D.U., Keesing, F., Lovell, C.R., Milne, B.T., Molles, M,C., Roberts, D.W., Strauss, S.Y.: Frontiers of ecology. Bioscience 51, 15-24 (2001)

2. Scheffer, M., Carpenter, S., Foley, J.A., Folke, C., Walker, B.: Catastrophic shifts in ecosystems. Nature 413, 591-596 (2001)

3. Levin, S.A.: The problem of pattern and scale in ecology. Ecology 73, 1943-1967 (1992)

4. Levin, S.A.: Ecosystems and the biosphere as complex adaptive systems. Ecosystems 1, 431-436 (1998)

5. Levin, S.A.: Fragile Dominion: Complexity and the Commons. Helix, Cambridge, MA (1999)

6. Holland, J.H.: Hidden Order: How Adaptation Builds Complexity. Helix, Reading, MA (1995)

7. Ulanowicz, R.E.: Complexity, stability and self-organization in natural communities. Oecologia (Berlin) 43, 295-298 (1979)

8. Rietkerk, M.G., Boerlijst, M., van Langevelde, F., HillerisLambers, R., van de Koppel, J., Kumar, L., Prins, H.H.T., De Roos, A.M.: Self-organization of vegetation in arid ecosystems. Am. Nat. 160, 524-530 (2002)

9. Van de Koppel, J., Van der Wal, D., Bakker, J.P., Herman, P.J.M.: Self organization and vegetation collapse in salt marsh ecosystems. Am. Nat. 165, E1-E12 (2005)

10. Wesenbeeck, V., Van de Koppel, J., Herman, P., Bouma, T.: Does scale-dependent feedback explain spatial complexity in salt-marsh ecosystems? Oikos 117, 152-159 (2008)

11. Neutel, A.M., Heesterbeek, J.A.P., Van de Koppel, J., Hoenderboom, G., Vos, A., Kaldeway, C., Berendse, F., de Ruiter, P.C.: Reconciling complexity with stability in naturally assembling food webs. Nature 449, 599-602 (2007)

12. Azza, N.K., van de Deeny, J.P., Kansiime, F.: Shoreline vegetation distribution in relation to wave exposure and bay characteristics in a tropical great lake, Lake Victoria. J. Trop. Ecol. 23, 353-360 (2007)

13. Temmerman, S., Bouma, T.J., Van de Koppel, J., Van der Wal, D., De Vries, M.B., Herman, P.M.J.: Vegetation causes channel erosion in a tidal landscape. Geology 35, 631-634 (2007)

14. Kuparinen, A., Snäll, T., Vänskä, S., O’Hara, R.B.: The role of modeling selection in describing stochastic ecological processes. Oikos 116, 966-974 (2007)

15. Azza, N. Denny, P., Van de Koppel, J., Kansiime, F.: Floating mats: their occurrence and influence on shoreline distribution of emergent vegetation. Freshw. Biol. 51, 1286-1297 (2006)

16. Gascoigne, J., Beadman, H.A., Saurel, C., Kaiser, M.J.: Density dependence, spatial scale and patterning in sessile biota. Oecologia 145, 371-381 (2005)

17. HilleRisLambers, R., Van de Koppel, J., Herman, P.M.J.: Persistence despite omnivory: benthic communities and the discrepancy between theory and observation. Oikos 113, 23-32 (2006)

18. Van de Koppel, J., Altieri, A.H., Silliman, B.R., Bruno, J.F., Bertness, M.D.: Scale-dependent interactions and community structure on cobble beaches. Ecol. Lett. 9, 45-50 (2006)

19. Silliman, B.R., Van de Koppel, J., Bertness, M.D., Stanton, L.E., Mendelssohn, I.A.: Drought, snails, and large-scale die-off of southern US salt marshes. Science 310, 1803-1806 (2005)

20. Van de Koppel, J., Bardgett, R.D., Bengtsson, J., Rodriguez-Barrueco, C., Rietkerk, M., Wassen, M.J., Wolters, V.: The effects of spatial scale on trophic interactions. Ecosystems 8, 801-807 (2005) 
21. Bouma, T.J., De Vries, M.B., Low, E., Peralta, G., Tanczos, C., Van de Koppel, J., Herman, P.M.J.: Tradeoffs related to ecosystem engineering: a case study on stiffness of emerging macrophytes. Ecology 86, 2187-2199 (2005)

22. Adema, E.B., Van de Koppel, J., Meijer, H.A.J., Grootjans, A.P.: Enhanced nitrogen loss may explain alternative stable states in dune slack succession. Oikos 109, 374-386 (2005)

23. Van de Koppel, J., Rietkerk, M., Dankers, M., Herman, P.M.J.: Scale-dependent feedback and regular spatial patterns in young mussel beds. Am. Nat. 165, E66-E77 (2005)

24. Rietkerk, M.G., Dekker, S.C., de Ruiter, P.C., Van de Koppel, J.: Self-organized patchiness and catastrophic shifts in ecosystems. Science 305, 1926-1929 (2004)

25. Bos, D., van de Koppel, J., Weissing, F.J.: Dark-bellied Brent geese aggregate to cope with increased levels of primary production. Oikos 107, 485-496 (2004)

26. van de Koppel, J., Rietkerk, M.: Herbivore regulation and irreversible vegetation change in semi-arid grazing systems. Oikos 90, 253-260 (2002)

27. Kumar, L., Rietkerk, M.G., Langevelde, F.V., van de Koppel, J., Andel, J.v., Hearne, J., de Ridder, N., Stroosnijder, L., Skidmore, A.K., Prins, H.H.T.: Relationship between vegetation growth rates at the onset of the wet season and soil type in the Sahel of Burkina Faso: implications for resource utilisation at large scales. Ecol. Model. 149, 143-152 (2002)

28. Alados, C.L., Navarro, T., Komac, B., Pascual, V., Martinez, F., Cabezudo, B., Pueyo, Y.: Do vegetation patch spatial patterns disrupt the spatial organization of plant species? Ecol. Complex. 6, 197-207 (2009)

29. Esteban, J., Fairén, V.: Self-organized formation of banded vegetation patterns in semi-arid regions: a model. Ecol. Complex. 3, 109-118 (2006)

30. Gillson, L.: Testing non-equilibrium theories in savannas: 1400 years of vegetation change in Tsavo National Park, Kenya. Ecol. Complex. 1, 281-298 (2004)

31. Somodi, I., Virágh, K., Aszalós, R.: The effect of the abandonment of grazing on the mosaic of vegetation patches in a temperate grassland area in Hungary. Ecol. Complex. 1, 177-189 (2004)

32. van de Koppel, J., Crain, C.M.: Scale-dependent inhibition drives regular tussock spacing in a freshwater marsh. Am. Nat. 168, E136-E147 (2006)

33. Garvie, M.R.: Finite-difference schemes for reaction-diffusion equations modelling predator-prey interactions in Matlab. Bull. Math. Biol. 69, 931-956 (2007)

34. Murray, J.D.: Mathematical Biology, 2nd edn. Biomathematics. Springer, Berlin (1993)

35. Andresn, P., Bache, M., Mosekilde, E.: Stationary space-periodic structures with equal diffusion coefficients. Phys. Rev. E 60, 297-301 (1999)

36. Henry, B.I., Wearne, S.L.: Existence of Turing instabilities in a two-species fractional reaction-diffusion system. SIAM J. Appl. Math. 62, 870-887 (2002)

37. Kuznetsov, S.P., Dewel, G., Borckmans, P., Mosekilde, E.: Absolute and convective instabilities in a one-dimensional Brusselator flow model. J. Chem. Phys. 106, 7609-7616 (1997)

38. Liu, Q.X., Jin, Z., Li, B.L.: Numerical investigation of spatial pattern in a vegetation model with feedback function. J. Theor. Biol. 254, 350-360 (2008)

39. Landau, L.D., Lifshitz, E.M.: Fluid Mechanics. Pergamon, Oxford (1959)

40. Briggs, R.J.: Electron Stream Interaction with Plasma. MIT, Cambidge (1964)

41. Bers, A.: Space - time evolution of plasma instabilities — absolute and convective. In: Rosenbluth, M.N., Sagdeev, R.Z. (eds.) Handbook of Plasma Physics, vol. 1. North-Holland, Amsterdam (1983)

42. Michailovsky, A.B.: Theory of Plasma Instabilities (Teorija Plazmennykh Neustoichivostei), vol. 1. Atomizdat, Moscow (1975)

43. Lifshitz, E.M., Pitaevskii, L.P.: Physical Kinetics. Pergamon, London (1981)

44. Deissler, R.J.: Noise-sustained structure, intermittency, and the Ginzburg-Landau equation. J. Stat. Phys. 40, 371-395 (1985)

45. Kuptsov, P.V.: Rigid transition to the stationary structure and imposed convective instability in a reactiondiffusion system with flow. Physica D 197, 174-195 (2004)

46. van Saarloos, W.: Front propagation into unstable states: marginal stability as a dynamical mechanism for velocity selection. Phys. Rev. A 37, 211-229 (1988)

47. van Saarloos, W.: Front propagation into unstable states. II. Linear versus nonlinear marginal stability and rate of convergence. Phys. Rev. A 39, 6367-6390 (1989)

48. Medvinsky, A.B., Petrovskii, S.V., Tikhonova, I.A., Malchow, H., Li, B.L.: Spatiotemporal complexity of plankton and fish dynamics. SIAM Rev. 44, 311-370 (2002)

49. Sherratt, J., Lewis, M.A., Fowler, A.C.: Ecological chaos in the wake of invasion. Proc. Natl. Acad. Sci. U. S. A. 92, 2524-2528 (1995)

50. Petrovskii, S.V., Malchow, H.: Wave of chaos: new mechanism of pattern formation in spatio-temporal population dynamics. Theor. Popul. Biol. 59, 157-174 (2001) 
51. Sun, G., Jin, Z., Liu, Q.X., Li, L.: Pattern formation in a spatial S-I model with non-linear incidence rates. J. Stat. Mech. 11, 11011 (2007)

52. Thomas, J.: Numerical Partial Differential Equations: Finite Difference Methods. Texts in Applied Mathematics, vol. 22. Springer, New York (1995)

53. Sun, G.Q., Jin, Z., Liu, Q.X., Li, L.: Pattern formation induced by cross-diffusion in a predator-prey system. Chin. Phys. B 17, 3936-3941 (2008)

54. Sun, G.Q., Jin, Z., Liu, Q.X., Li, L.: Spatial pattern in an epidemic system with cross-diffusion of the susceptible. J. Biol. Syst. 17, 141-152 (2009)

55. Wang, R.H., Liu, Q.X., Sun, G.Q., Jin, Z., van de Koppel, J.: Nonlinear dynamic and pattern bifurcations in a model for spatial patterns in young mussel beds. J. R. Soc. Interface (2009, in press)

56. Sun, G.Q., Jin, Z., Liu, Q.X., Li, L.: Chaos induced by breakup of waves in a spatial epidemic model with nonlinear incidence rate. J. Stat. Mech. 2008, P08011 (2008)

57. Doedel, E.J., Paffenroth, R.C., Champneys, A.R., Fairgrieve, T.F., Kuznetsov, Y.A., Oldeman, B.E., Sandstede, B., Wang, X.: AUTO2000: continuation and bifurcation software for ordinary differential equations (with HomCont). Technical Report, Caltech (2001)

58. Apfeelbaum, S.I., Leach, M., Baller, R.: Restor. Manage. Notes 7, 38 (1989)

59. Cayouette, J., Catling, P.M.: Hybridization in the genus Carex with special reference to North America Bot. Rev. 58, 351-438 (1992)

60. Rietkerk, M., van de Koppel, J.: Regular pattern formation in real ecosystems. Trends Ecol. Evol. 23, $169-175(2008)$ 